\title{
Sorption of the nematicide fluensulfone in six UK arable soils - implications for control of the potato cyst nematode Globodera pallida
}

by Norshie, P.M., Grove, I.G. and Back, M.A.

Copyright, Publisher and Additional Information: This is the author accepted manuscript. The final published version (version of record) is available online via Brill Online Please refer to any applicable terms of use of the publisher.

DOI: $\underline{0.1163 / 15685411-00003128}$

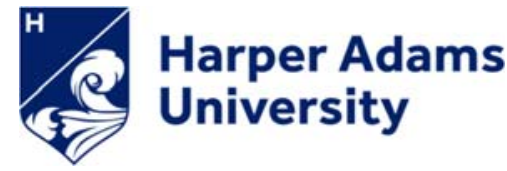

Norshie, P.M., Grove, I.G. and Back, M.A. 2017. Sorption of the nematicide fluensulfone in six UK arable soils - implications for control of the potato cyst nematode Globodera pallida. Nematology. 
1 Title: Sorption of the nematicide fluensulfone in six UK arable soils - implications for control of

2 the potato cyst nematode Globodera pallida

5 Running title: Sorption of nematicide fluensulfone in UK arable soils

6

$8 \quad$ Patrick M Norshie, ${ }^{a b *}$ Ivan G Grove ${ }^{b}$ and Matthew A Back ${ }^{b}$

$11 a b^{*}$ Department of Agronomy, University College of Agriculture and Environmental Studies, P.O.

12 Box 27, Bunso, Eastern Region, Ghana.

13

14 b Nematology and Entomology Group, Crop and Environment Research Centre, Harper Adams

15 University, Newport, Shropshire, TF10 8NB, UK.

16

17

18

19

20

$21{ }^{*}$ Corresponding to: PM Norshie, P. O. Box AN 18095, Accra-North, Accra, Ghana

22 Ghana. E-mail: norshie@yahoo.com. 
1 Summary - Batch adsorption experiments were performed to determine the sorption of the

2 nematicide fluensulfone in the technical-grade form and granular formulation (as Nimitz $15 \mathrm{G}^{\circledR}$ ) in

3 six UK arable soils. The Freundlich and equilibrium sorption coefficients $K_{\mathrm{F}}$ and $K_{\mathrm{D}}$, respectively,

$4 \quad$ were generally low. $K_{\mathrm{F}}$ and $K_{\mathrm{D}}$ correlated positively with soil organic matter in all instances. The

5 sorption kinetics were similar for both forms, but the $K_{\mathrm{D}}$ was about four times lower for Nimitz

$6 \quad 15 \mathrm{G}$ than the technical-grade, suggesting concentration dependency of fluensulfone sorption. The

7 low sorption of fluensulfone across the soils, indicates that partitioning of fluensulfone to the soil

8 liquid phase may be unlimited. Therefore, substantial availability in the soil to be effective is likely.

9 Sorption, therefore, may not limit fluensulfone efficacy. Nonetheless, these results call for cautious 10 use of the nematicide because leaching is possible.

11

12

13

14

15

16

17

18

19

20

21 Keywords - Adsorption; efficacy; Globodera species; Nimitz

22 Sorption is a key factor which influences a pesticide's environmental fate and biological activities

23 (Kravvariti et al., 2010; Wang \& Keller, 2009; Villaverde et al., 2008). Sorption may involve both 
1 adsorption processes representing uptake of molecules onto the outer surfaces of soil particles

2 (Calvet, 1989) and absorption processes where a pesticide permeates the soil matrix (Thompson.

3 \& Gone, 2012). Irrespective of the form in which it manifests, sorption entails the removal of a

4 pesticide from the soil solution (Sposito, 2008), and could constitute a limiting factor to nematicide

5 efficacy (Smelt \& Leistra, 1992) since nematodes are essentially aquatic organisms that exist in

6 the soil water.

7 Fluensulfone is the active ingredient in $N i m i t z \circledR$ a recent non-fumigant and contact nematicide

8 from ADAMA Agricultural Solutions Ltd, and is currently registered for use as a pre-planting

9 treatment to solanaceous and cucurbitaceous crops in USA, Canada, Mexico, Dominican Rep.,

10 Panama, Israel, Japan, Taiwan, S. Korea, and Australia Danny Karmon, Senior Innovative Product

11 Manager, ADAMA Agricultural Solutions Ltd. Israel. Personal Communication). Fluensulfone is

12 favoured to the organophosphate fosthiazate and the carbamate oxamyl nematicides, for its lesser

13 acute toxicity for oral ingestion, dermal contact and inhalation, and is deemed to have low

14 toxicological activity on birds, fish, and bees (Danny Karmon, Senior Innovative Product

15 Manager, ADAMA Agricultural Solutions Ltd. Israel. Personal Communication). The activities

16 of fluensulfone have been reported on root-knot nematode, Meloidogyne species (Oka et al., 2009,

17 2012, 2013; Morris et al., 2015), migratory nematodes, Pratylenchus penetrans, P. thornei and

18 Xiphinema index (Oka, 2014), and the potato cyst nematode Globodera pallida (Norshie et al.,

19 2016). The mode of action is reported to involve cessation of feeding, paralysis and complete death

20 (Kearn et al., 2017).

Fluensulfone is a fluorinated molecule, and the influence of fluorine on a molecule's polarity

22 is known to involve improvement of a molecule's lipophilicity (Dinoiu, 2006; Ichino et al., 1990),

23 and allows changes towards non-polarity (Smart, 2001). With fluensulfone deemed to be 
1 lipophilic, it is presumed that its sorption to a soil shall depend mainly on the soil's organic matter

2 content. A direct relationship between soil organic peat amendments and fluensulfone efficacy for

3 control of Meloidogyne spp. on tomato has been reported by Oka et al., (2013). Yet, fluensulfone

4 sorption in soil remains to be measured. The experiments reported in this paper were part of a

5 broad study which evaluated the efficacy of fluensulfone in the management G. pallida. The

6 objectives were to (i) determine the sorption of fluensulfone as a function of soil type (ii) compare

7 the sorption of the technical-grade form to that of the granular formulation (as Nimitz $15 \mathrm{G}^{\circledR}$ ), and

8 (iii) determine the sorption of fluensulfone in soil following organic treatment.

\section{$9 \quad$ Materials and methods}

11 SUBSTRATES STUDIED

12 The soils investigated (Table 1) were collected from the top $0-30 \mathrm{~cm}$ depth of commercial potato 13 fields in Cambridgeshire, Lincolnshire, Nottinghamshire and Shropshire in England between June 142010 and May 2011. These fields were used to evaluate fluensulfone (Nimitz 15G) for 15 management of G. pallida. The soils were used after drying for, at least, seven days at a temperature of $20{ }^{\circ} \mathrm{C}$, and sieving through a 2-mm aperture sieve (Endecotts Ltd. London,

17 England). Moss peat was purchased from Clover Peat Products (Dungannon, Northern Ireland), 18 sieved to $<2 \mathrm{~mm}$ size, and used as an organic amendment. The soils were analysed for $\mathrm{pH}$, residual 19 water content and texture by standard methods (MAFF, 1985; 1986). Soil organic carbon (SOC) 20 content was determined using a LECO sulphur-carbon analyser $\left(\right.$ LECO $^{\circledR}$ Hazer Grove, Stockport,

21 UK). The soils' water-holding capacity (WHC) was determined using a model 1600 Pressure Plate 22 Extractor (ELE International, Bedfordshire, UK). 
CHEMICALS AND REAGENTS

2 ADAMA Agricultural Solutions Ltd. supplied fluensulfone (a technical-grade of $>95 \%$ purity and

3 Nimitz $\left.15 \mathrm{G}^{\circledR}\right)$ for the study. Fluensulfone is moderately soluble in water $\left(622 \mathrm{mg} / 1\right.$ at $\left.20^{\circ} \mathrm{C}\right)$, has a

$4 \log P_{\text {ow }}$ at $\mathrm{pH} 7.5$ of 2.6 , and $3.1 \times 10^{-3} \mathrm{~Pa}$ vapour pressure. Stock and working fluensulfone

5 solutions were prepared in $0.01 \mathrm{M}$ calcium chloride $\left(\mathrm{CaCl}_{2}\right)$ (Sigma-Aldrich Limited, UK) in

6 deionised water. Acetonitrile (99.99\% purity) and Orthophosphoric acid (85\% purity) were

7 purchased from Fisher Scientific Ltd and BDH Laboratory supplies UK Ltd, respectively.

9 GENERAL METHODS

10 The sorption of fluensulfone was determined by the batch equilibrium method following the 11 Organization for Economic Co-operation and Development (OECD) guideline 106 (OECD, 2000).

12 Soil suspensions (10 $\mathrm{mg}$ soil in $10 \mathrm{ml}$ of $0.01 \mathrm{M} \mathrm{CaCl}_{2}$ ), were prepared and pre-equilibrated by 13 agitating for $16 \mathrm{~h}$ on a HS 501 digital reciprocal shaker (IKA ${ }^{\circledR}$ Werke GmbH \& Co. KG, Staufen, 14 Germany). All experiments were conducted at a temperature of $20{ }^{\circ} \mathrm{C}$. The suspensions were 15 centrifuged using Eppendorf Centrifuge 5810 (Eppendorf AG, Hamburg, Germany). Supernatants 16 were sampled with $2 \mathrm{ml}$ syringes (BD Plastics Ltd, Sunderland, UK), whenever appropriate, and 17 were sieved through $0.2 \mu \mathrm{m}$ pore size Polyvinylidene Difluoride (PVDF) syringe filters (GE 18 Healthcare Ltd, Hertfordshire, UK) prior to analysis for fluensulfone. Extracts were analysed 19 immediately or stored at $-20^{\circ} \mathrm{C}$ for future analysis. Fluensulfone was quantified by high pressure 20 liquid chromatography (HPLC) analysis following conditions reported by Norshie et al., (2017). 21 
2 Sorption isotherms were determined in two separate experiments (Experiment 1 \& 2), each conducted three times at four initial concentrations $\left(C_{\mathrm{I}}\right)$ of $1.25,2.50,5.00$ and $7.50 \mathrm{mg} / 1$.

4 Experiment 1 utilised the six original soils whereas Experiment 2 was conducted using Soil HS

5 (See Table 1) following treatments with moss peat at five levels (0.0, 1.2, 2.4, 4.8 and 9.6\%). Soil

6 HS was selected for Experiment 2 because it gave the highest scorpion in Experiment 1, and had

7 been used in a pot efficacy experiment. After pre-equilibration, soil suspensions $(1 / 1 \mathrm{w} / \mathrm{v}) \mathrm{were}$

8 spiked with an appropriate volume of $50 \mathrm{mg} / 1$ stock fluensulfone to give the required $C_{\mathrm{I}}$. After

9 shaking for $2 \mathrm{~h}$ (shown in a preliminary experiment to give equilibrium concentration $C_{\mathrm{E}}$; data not

10 shown), the suspensions were centrifuged at $3000 \mathrm{~g}$ for $15 \mathrm{~min}$ and a $1 \mathrm{ml}$ aliquot was analysed for

11 the $C_{\mathrm{E}}$. The proportion of fluensulfone sorbed $\left(C_{\mathrm{S}}\right)$ was determined as the difference between $C_{\mathrm{I}}$

12 and $C_{\mathrm{E}}$. Sorption isotherms were described by fitting the linear form of Freundlich sorption model

13 to $C_{\mathrm{S}}$ and $C_{\mathrm{E}}$ according to the equation in (1), where, $K_{\mathrm{F}}$ and $1 / n$ respectively are the Freundlich

14 sorption coefficient and sorption exponent. These parameters were determined by regressing Log

$15 C_{\mathrm{S}}$ against $\log C_{\mathrm{E}}$, with $K_{\mathrm{F}}$ and $1 / n$, respectively, represented by the intercept and slope of the 16 regression equation. Sorption was normalised to soil organic carbon $\left(K_{\mathrm{FOC}}\right)$ using the equation 17 shown in (2), where OC is the organic carbon contents of soil.

$$
\log (C s)=\log \left(K_{\mathrm{F}}\right)+1 / n \log \left(C_{\mathrm{E}}\right)
$$

$$
K_{\mathrm{FOC}}=K_{\mathrm{F}} \times 100 / \mathrm{OC}
$$

22 This experiment was conducted to compare the sorption of technical-grade fluensulfone with 23 Nimitz $15 \mathrm{G}^{\circledR}$ as per sorption coefficient $\left(K_{\mathrm{D}}\right)$. The experiment was conducted three times utilising 
1 the original soils with the exception of Soil GL. After pre-equilibration, the suspensions $(1 / 1 \mathrm{w} / \mathrm{v})$

2 were either spiked with $1 \mathrm{~mL}$ of $50 \mathrm{mg} \mathrm{m} / 1$ stock fluensulfone or were treated with $34 \mathrm{mg}$ of

3 granules. The samples were shaken for 2, 4, 6, and $8 \mathrm{~h}$, when the liquid phase fluensulfone was

4 quantified. Fluensulfone that was sorbed $(C s)$, as percentage of the $C_{\mathrm{I}}$, was determined according

5 to equation (3). $K_{\mathrm{D}}$ was calculated by the equation in (4). In addition to the test suspensions, the

6 setup included two additional samples, both without soil, one of $9 \mathrm{ml} \mathrm{CaCl}_{2}$ spiked with $1 \mathrm{~mL}$ of

$750 \mathrm{mg} / \mathrm{l}$, the other of $10 \mathrm{ml} \mathrm{CaCl} 2$ treated with $34 \mathrm{mg}$ of Fluensulfone $15 \mathrm{G}$. These were used to

8 determine solution concentration of fluensulfone within the period of the experiment, and use to

9 correct for sorption estimates.

$$
K_{\mathrm{D}}=C_{\mathrm{S}} / C_{\mathrm{E}}
$$

12 where, $C_{\mathrm{T}}$ is the concentration measured at the respective sampling times.

13

\section{EXPERIMENTAL DESIGN AND ANALYSIS OF DATA}

15 The experiments were a randomised block design where each cycle of experiment was treated as 16 a replicate. The data was subjected to analysis of variance in GenStat for Windows ${ }^{\circledR}$ V.15 (VSN

17 International Ltd. Hempstead, UK). Differences between means were compared as per Fisher's 18 protected LSD post hoc tests at $P=0.05$.

\section{Results}


2 Figure 1 shows the isotherms obtained for fluensulfone sorption in the six original soils Fig. 1A) and the peat-amended Soil HS (Fig. 1B). The Freundlich sorption parameters $\left(K_{\mathrm{F}}\right.$ and $\left.1 / n\right)$, the

4 coefficients of determination $\left(r^{2}\right)$ and sorption normalised to Soil OC $\left(K_{\mathrm{FOC}}\right)$ are presented in Table 2. The isotherms for the original and peat-treated soils were nonlinear S-type $(1 / n>1)$ according to Giles' classification (Giles, 1960). The $K_{\mathrm{F}}$ was lowest in Soil MN and highest in Soil HS $(P<$

7 0.001). A similar trend was observed for the parameter KFOC. A positive correlation $\left(r^{2}=0.907 ; P\right.$

$8=0.013$ ) was found between $K_{\mathrm{F}}$ and soil OC (Table 3). Good, but non-significant correlations were, 9 also, seen between $K_{\mathrm{F}}$ and soil clay $\left(r^{2}=0.554 ; P=0.256\right)$ and soil $\mathrm{pH}\left(r^{2}=-0.744 ; P=0.09\right)$. The peat amendments applied to Soil HS had affected parameters $K_{\mathrm{F}}$ and $1 / n$ (Table 2), both of which, correlated positively with Soil OC but significantly only $\left(r^{2}=0.88 ; P=0.05\right)$ for the technical-grade (Table 4). $1 / n$ was inversely, but not significantly $\left(r^{2}=-0.777 ; P=0.123\right)$, related to the amendments with the $S$-type isotherm, describing sorption in the original Soil HS (moss peat 14 at $0.0 \%$ ), being transformed to an L-type isotherm $(1 / n<1)$ at the $2.4,4.8$ and $9.6 \%$ amendments not shown). The $K_{\mathrm{DS}}$ are plotted against the peat treatments in Fig. 3; significantly positive correlations were found between $K_{\mathrm{D}}$ for both formulations of the product and the treatments $\left(r^{2}=\right.$ 24 significantly greater $(P<0.001)$ after the technical-grade than Nimitz $15 \mathrm{G}$. 


\section{EFFECTS OF GRANULAR FORMULATION ON FLUENSULFONE SORPTION}

3 Figure 4 shows sorption kinetics over an eight-hour period for the the technical-grade and Nimitz

$4 \quad 15 \mathrm{G}$. Sorption of both forms followed similar kinetics, reaching equilibrium within $2 \mathrm{~h}$. This was

5 in agreement with preliminary test results (Data not shown). Sorption after the formulation was

6 lower $(P<0.001)$ than the technical-grade. The sorption estimates were corrected, accordingly,

7 for these differences. The $K_{D}$ values obtained are presented in Fig. 5. Regardless of soil type, the

$8 K_{\mathrm{D}}$ was higher $(P<0.001)$ for the technical-grade form $\left(\right.$ Mean $K_{\mathrm{D}}=1.37 \mathrm{mg} / \mathrm{kg}$ soil $)$ than the

9 formulation (Mean $K_{\mathrm{D}}=0.32 \mathrm{mg} / \mathrm{kg}$ soil). The soil effects were, also, highly significant $(P<$

0.001); $K_{\mathrm{D}}$ was lowest in Soil $\mathrm{MN}$ and highest in Soil WL. Positive correlations were found

11 between $K_{\mathrm{D}}$ and soil OC (Table 4), but marginally significant just after the technical grade form

$12 \quad\left(r^{2}=0.88 ; P=0.05\right)$.

\section{Discussion}

15 The results show a two-phase uptake of fluensulfone, with sorption occurring more within the first

16 two hours of making contact with the soils. This would imply a rapid removal from solution, in

17 which case, sorption may become a limiting factor to availability in the soil. On the other hand,

18 this rapid uptake could result in a greater retention of fluensulfone in the soil and could guard

19 against leaching and degradation. Two-phase sorption has been frequently reported in the literature

20 (Boesten, 1990; Ball \& Roberts, 1991). The initially fast phase has been ascribed to adsorption

21 processes (Cancela et al., 1990), and the slower phase is thought to be due to slow intra-particle

22 diffusion of molecules (White et al., 1986). If sorption of fluensulfone on the soils were by 23 adsorption, which accumulates molecules at the soil-liquid interface (Huang et al., 1996), then 24 even when sorbed, fluensulfone may be easily accessible to percolating soil water, and perhaps, 
be readily available for distribution in the soil. In this case, an adsorption driven sorption could

2 concentrate fluensulfone around soil particles thereby exposing second-stage G. pallida juveniles

3 to a high dosage. On the other hand, however, greater availability to soil water may suggest

4 vulnerability to leaching agents. Furthermore, it has been suggested (Barbercheck \& Duncan,

5 2004) that aerobic organisms and activity are, mostly, associated with outer surfaces of soil

6 particles. If fluensulfone is predominantly adsorbed, it could as well be that its persistence may be

7 shortened by enhanced availability to microbial degraders.

Sorption of molecules, exhibiting low water solubility, has been suggested to occur mainly by

9 hydrophobic partitioning into soil organic matter (Koenning \& Sipes, 1998). This often, yields

10 linear isotherms (Scow \& Johnson, 1997). Even though the sorption of fluensulfone depended

11 mainly on the soil organic matter, the isotherms observed were essentially nonlinear $(1 / n \neq 1)$. This

12 may indicate that the sorption observed was not exclusively influenced by hydrophobic

13 partitioning. An S-type isotherm denotes more rapid sorption as solute concentration increases

14 (Murphy et al., 1990), and is characteristic of sorption of organic compounds onto a surface with

15 low sorbing potential (Chiou et al., 1998). This condition typically relates to the adsorption of

16 organic compounds onto clay surfaces (Delle Site, 2001; Sposito, 1984). Therefore, the

17 involvement of soil clay in the sorption of fluensulfone is likely. The transformation of the S-type isotherm, describing sorption in the original soil HS, to an L-type isotherm following the peat

19 treatments of this soil provided even greater evidence of uptake by the soil's clay fraction. It is

20 possible that the addition of peat may have had a diminishing effect on the contribution of clay to

21 the sorption process; this could be expected as the proportion of clay in the soils is diluted with the

22 increasing addition of peat. The suggestion, therefore, is that sorption of fluensulfone may be due

23 to hydrophobic partitioning, but also by adsorption onto the mineral fraction, mainly clay. 
The sorption of fluensulfone varied widely between the original soils, but was generally, low across the soils. This could mean low sorbing potentials for fluensulfone, at least in the soil studied here. The correlation between fluensulfone sorption and the soils' organic matter content highlights the importance of the molecules hydrophobicity in its affinity for soil. Inferences from the selected properties would suggest moderate hydrophobicity, and could relate to the low sorption across the soils. Indeed, treating Soil HS with peat significantly enhanced this soil's capacity for sorbing fluensulfone. Even then, it is worth noting that the increases in sorption did not commensurate with the levels of the peat treatment, and was a further indication of potentially low affinity of fluensulfone for the soils. Being lowly sorbed implies that substantial proportions of fluensulfone would partition to the soil liquid phase for distribution and nematicidal activities. Furthermore, the generally low $K_{F O C}$ indicates that fluensulfone may be mobile in the soil, and as such, may be highly dispersible in percolating soil water. The low sorption of fluensulfone in soil indicates that it has greater potential for controlling G. pallida in a wide range of soils.

The difference in sorption of the technical-grade and the granular product is attributable to limited availability of fluensulfone in the solution for uptake. Indeed, a granular formulation releases active substances gradually (Kile et al., 1999) and, was evident for Nimitz 15G (Norshie et al., 2017). The lower sorption exhibited here would suggest that studies involving the technicalgrade could over-estimate sorption in potato beds receiving a treatment of this formulation. Coupling effects of limited availability and fast sorption further suggest that low soil solution concentrations could be expected when Nimitz $15 \mathrm{G}$ is applied to potato beds. Furthermore, the diminishing effect on sorption implies plausible limiting effects on degradation, as well as leaching of the molecules in potato beds. 
The results have shown low sorption of fluensulfone in a range of soils, suggesting that the nematicide will be readily available in the soil's aqueous phase for effective control of G. pallida. These results, however, indicate that fluensulfone may be easily leached. Even though this highlights environmental consequences, our study on persistence in field soil (Norshie et al., 2017) indicated that it may not pose a hazard to the environment.

\section{Acknowledgements}

The authors thank Mr Kevin Jones and Mr Amjad Ali for technical support during the HPLC analysis. The first author was in receipt of a PhD studentship from ADAMA Agricultural Solution Ltd.

\section{References}

Ball, W.P. \& Roberts, P.V. (1991). Long-term sorption of halogenated organic chemicals by aquifer material. 1. Equilibrium. Environment Science and Technology 25:1223-1237. DOI: 10.1021/es00019a002

Barbercheck, M.E \& Duncan, L. (2004). Abiotic factors In: R. Gaugler and A. L. Bilgrani (eds.) Nematode behaviour. CAB International, pp. 309-343.

Boesten, J.J. (1990). Influence of solid/liquid ratio on the experimental error of sorption coefficients in pesticide/soil systems. Pesticide Science 30:31-41. DOI: $10.1002 /$ ps. 2780300105 
1 Calvet, R. (1989). Adsorption of organic chemicals in soils. Environmental Health Perspectives $83: 145-177$.

Cancela, G.D., Taboada, E.R. \& Sanchez-Rasero, F. (1990). Adsorption of Cyanazine on Peat and Montmorillonite Clay Surfaces1. Soil Science 150:836-843.

5

Delle Site, A. (2001). Factors affecting sorption of organic compounds in natural sorbent/water systems and sorption coefficients for selected pollutants. A review. Journal of Physical and Chemical Reference Data 30:187-439. DOI: dx.doi.org/10.1063/1.1347984

Dinoiu, V. (2006). Fluorine Chemistry: Past, Present and Future Review. Roumaine de Chimie 51: $1141-1152$.

Giles, C.H., MacEwan, T., Nakhwa, S. \& Smith, D. (1960). Studies in adsorption. Part XI. A system of classification of solution adsorption isotherms, and its use in diagnosis of adsorption mechanisms and in measurement of specific surface areas of solids. Journal of the Chemical Society (Resumed) 0:3973-3993. DOI: 10.1039/JR9600003973

Huang, W., Schlautman, M.A. \& Weber, W.J. (1996). A distributed reactivity model for sorption by soils and sediments. 5. The influence of near-surface characteristics in mineral domains. Environment Science and Technology 30:2993-3000. DOI: 10.1021/es960029w 
1 Ichino, T., Sasaki, S., Matsuura, T. \& Nishi, S. (1990). Synthesis and properties of new polyimides containing flourinated alkoxy side chains. Journal of Polymer Science Part A: Polymer Chemistry 28:323-331. DOI: 10.1002/pola.1990.080280208

4 Kile, D.E., Wershaw, R.L \& Chiou, C.T. (1999). Correlation of soil and sediment organic matter 5

Koenning, S.R. \& Sipes, B.S. (1998). Biology. In: S.B. Sharma, (ed.), The Cyst nematode. Kluwer Academic Publishers, Great Britain. pp. 156-190.

9 Kravvariti, K., Tsiropoulos, N.G. \& Karpouzas, D.G. (2010). Degradation and adsorption of 10 terbuthylazine and chlorpyrifos in biobed biomixtures from composted cotton crop residues. Pest Management Science 66:1122-1128. DOI: 10.1002/ps.1990

12 Ministry of Agricultural, Fisheries and Food (MAFF), (1985). Soil Texture System and pesticide 13 use. Pamphlet 3001.

14 Ministry of agriculture, fisheries \& food (MAFF), (1986). The analysis of agricultural materials, 15 $3^{\text {rd }}$ edition. Reference book 427. Her Majesty's office, London, UK. of Fluensulfone in a Tomato-Cucumber Double Cropping System. Journal of Nematology 47:310-315. 
1 Murphy, E.M., Zachara, J.M. \& Smith, S.C. (1990). Influence of mineral-bound humic substances on the sorption of hydrophobic organic compounds. Environment Science and Technology 24:1507-1516. DOI: 10.1021/es00080a009

4 Norshie, P.M., Grove, I.G. \& Back, M.A. (2016). Field evaluation of the nematicide fluensulfone for control of the potato cyst nematode Globodera pallida. Pest Management Science DOI: $10.1002 /$ ps. 4329

Norshie, P.M., Grove, I.G. \& Back, M.A. (2017). Persistence of the nematicide fluensulfone in potato (Solanum tuberosum ssp. tuberosum) beds under field conditions. Nematology 19: 739-747. DOI: $10.1163 / 15685411-00003085$

OECD Guidelines for the testing of chemicals (2000). Test No. 106: adsorption-desorption using

a batch equilibrium method. OECD Organisation for Economic Co-operation and Development. Available online at http://www.oecd-ilibrary.org. Retrieved on 02 July, 2011. 
1 Oka, Y., Shuker, S. \& Tkachi, N. (2012). Systemic nematicidal activity of fluensulfone against the root-knot nematode Meloidogyne incognita on pepper. Pest Management Science 68:268275. DOI: $10.1002 /$ ps.2256

4 Oka, Y., Shuker, S. \& Tkachi, N. (2013). Influence of soil environments on nematicidal activity of fluensulfone against Meloidogyne javanica. Pest Management Science 69:1225-1234. DOI: $10.1002 /$ ps.3487

7 Scow, K.M. \& Johnson, C.R. (1997). Effect of Sorption on Biodegradation of Soil Pollutants. In: Advances in Agronomy Anonymous Academic Press, p. 1-56. 